\title{
3D BLOB BASED BRAIN TUMOR DETECTION AND SEGMENTATION IN MR IMAGES
}

\author{
Chen-Ping Yu ${ }^{1}$, Guilherme Ruppert ${ }^{4}$, Robert Collins ${ }^{2}$, Dan Nguyen $^{3}$, Alexandre Falcao ${ }^{4}$, Yanxi Liu ${ }^{2}$ \\ ${ }^{1}$ Dept. of Computer Science, Stony Brook University, Stony Brook, NY, USA \\ ${ }^{2}$ Dept. of Computer Science and Engineering and Dept. of Electrical Engineering, \\ Pennsylvania State University, University Park, PA, USA \\ ${ }^{3}$ Dept. of Radiology and Neurosurgery, The Milton S. Hershey Medical Center, Hershey, PA, USA \\ ${ }^{4}$ Institute of Computing, University of Campinas, Brazil
}

\begin{abstract}
Automatic detection and segmentation of brain tumors in 3D MR neuroimages can significantly aid early diagnosis, surgical planning, and follow-up assessment. However, due to diverse location and varying size, primary and metastatic tumors present substantial challenges for detection. We present a fully automatic, unsupervised algorithm that can detect single and multiple tumors from 3 to $28,079 \mathrm{~mm}^{3}$ in volume. Using 20 clinical 3D MR scans containing from 1 to 15 tumors per scan, the proposed approach achieves between $87.84 \%$ and $95.30 \%$ detection rate and an average end-to-end running time of under 3 minutes. In addition, 5 normal clinical 3D MR scans are evaluated quantitatively to demonstrate that the approach has the potential to discriminate between abnormal and normal brains.
\end{abstract}

Index Terms - brain tumor detection, MRI brain asymmetry, 3D separable Laplacian of Gaussian, 3D blob detection

\section{INTRODUCTION}

Accurate and fast brain tumor detection and segmentation from 3D MR images has become one of the necessary steps for computer aided diagnosis (CAD) in neuroradiology. Detection of multiple, small-sized tumors is of particular importance since this may lead to diagnosis of early stage tumors or may be indicative of potential metastasis.

The majority of the recent work in this area focuses on supervised learning methods for single tumor segmentation [3][4][5][6][7]. Supervised methods require excessive computation time for training on fully registered 3D brain scans, and a detailed comparison of prior work shows that automatic tumor segmentation can take up to hours per 3D scan [3]. Moreover, detection rates for various tumor sizes are not reported categorically. Some newer works address the detection of small (early stage) and multiple tumors (metastasis), and take advantage of the bilateral symmetry of the brain [8][9][10][11][12]. However, [12] processes the MR images slice by slice in $2 \mathrm{D},[8]$ only uses simulated tumors (7 cases) for evaluation, [11] only deals with 2D slices where a
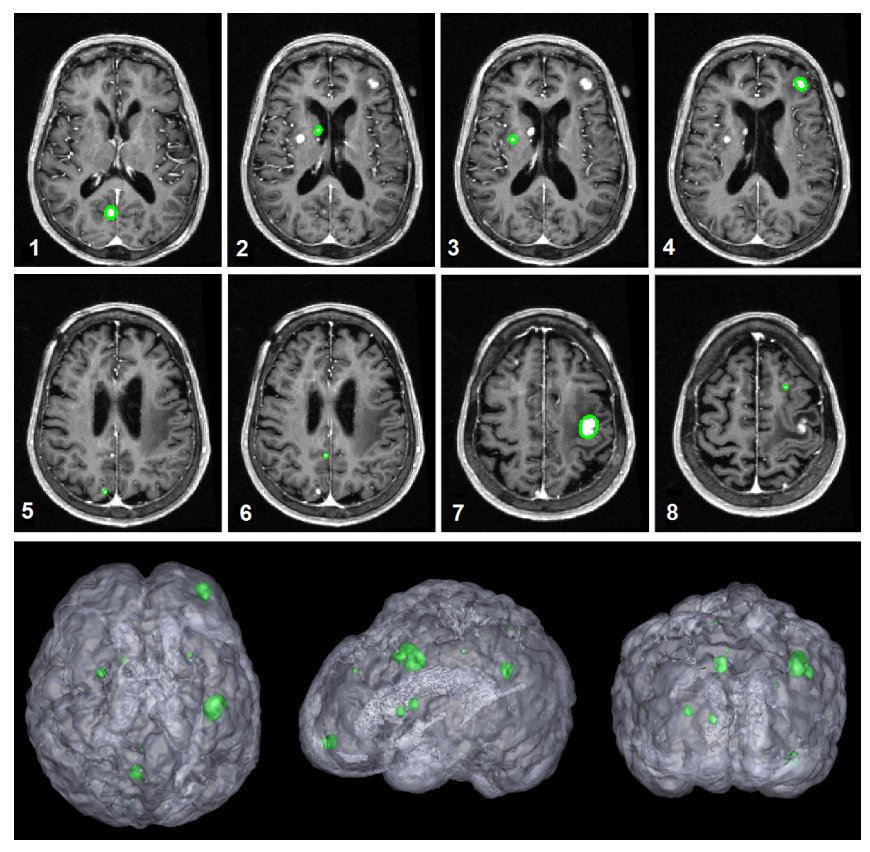

Fig. 1. Our detection results for case \#11 (8 tumors, Table 1). Row 1 and 2: the 8 detected tumors (circled in green); row 3: subsequent 3D segmentation result using IFT-Watershed [1], best viewed in color (visualized using ITK-SNAP [2]).

tumor already presents, and the smallest tumor sizes reported in [9][10] are 9 and $14 \mathrm{~mm}^{3}$ respectively, while ours is $3 \mathrm{~mm}^{3}$ (Table 11. The true positive over false positive ratio $T_{p}: F_{p}$ in [10] is approximately 1:10 versus 1:2.7 per brain in our case. Fig. 1 shows a sample result of our detection and segmentation process (\#11, Table 1) where all 8 tumors are found.

There are three technical contributions in this work: (1) an automated brain tumor detection algorithm for clinical 3D MR images; (2) a novel unsupervised sequential-pruning framework based on brain asymmetry and compactness of 3D blobs, and that bypasses full-brain registration, leading to the fastest reported running times (under 3 minutes per brain image) and best tumor detection rates $(87.84 \% \sim 95.30 \%)$ 
on the most challenging brain tumor image set reported thus far; and, (3) a fully automatic 3D tumor segmentation method using detected 3D blobs as initial seeds.

\section{PROPOSED METHOD}

Given a 3D MR brain scan, our algorithm extracts 3D blobs as potential regions-of-interest for tumor detection. To construct a computational basis for brain asymmetry analysis, we first apply a fully automatic midsagittal plane (MSP) extraction algorithm [13] and reorient the brain scan in alignment to the MSP. This is followed by automatic skull-stripping using the FMRIB Software Library (FSL) [14] to discard 3D blobs that are detected outside of the brain.

We compute a blob saliency response $(B)$, blob 3D shape compactness score $(S)$, and a quantified measure for bilateral brain asymmetry $(A)$ of each blob. These three feature scores are also combined to determine a tumor confidence $C$ :

$$
C(B, S, A)=\frac{B+A}{S}
$$

which is indicative of the likelihood of a given 3D blob being a tumor. This score is based on the observation that most brain tumors appear to be compact, blob-like objects that are bilaterally asymmetrical (located in only one hemisphere of the brain) [15][11]. Starting with a large initial set of extracted blobs, a sequential cascade of pruning steps based on shape, symmetry and tumor likelihood are performed (Figure 2) to remove false positives and isolate a small set of high-quality tumor hypotheses.

\subsection{D Laplacian of Gaussian (LoG) filtering}

Given that tumors are usually "blob-like" entities, we propose to use blob detection to automatically generate a pool of tumor candidates from 3D MR images. We use the Laplacian of Gaussian (LoG) filter as a general purpose 3D blob detector, leveraging its $1 \mathrm{D}$ separable form for fast computation:

$$
\begin{aligned}
& {\left[\left(f \otimes \Delta g_{x}\right) \otimes g_{y}\right] \otimes g_{z}+} \\
h(x, y, z \mid \sigma)= & {\left[\left(f \otimes \Delta g_{y}\right) \otimes g_{x}\right] \otimes g_{z}+} \\
& {\left[\left(f \otimes \Delta g_{z}\right) \otimes g_{x}\right] \otimes g_{y} }
\end{aligned}
$$

where $h$ is the LoG-filtered volumetric image, and $\Delta g$ and $g$ are 1D LoG and Gaussian filters, respectively. This formulation significantly reduces the multiplications per voxel of computing 3D LoG from $n^{3}$ to just $9 n$ (where $n$ is the number of voxels in the filter support region) and makes generalpurpose $3 \mathrm{D}$ blob detection in volumetric data feasible.

To find the radius of a 3D blob that corresponds to a given scale expressed by a scale parameter $\sigma$, we calculate the zerocrossing of the 3D isotropic LoG in polar coordinates:

$$
\Delta g_{x y z}=\frac{1}{\sigma^{5} 2 \pi \sqrt{2 \pi}}\left(\frac{r^{2}}{\sigma^{2}}-3\right) e^{\frac{-r^{2}}{2 \sigma^{2}}}
$$

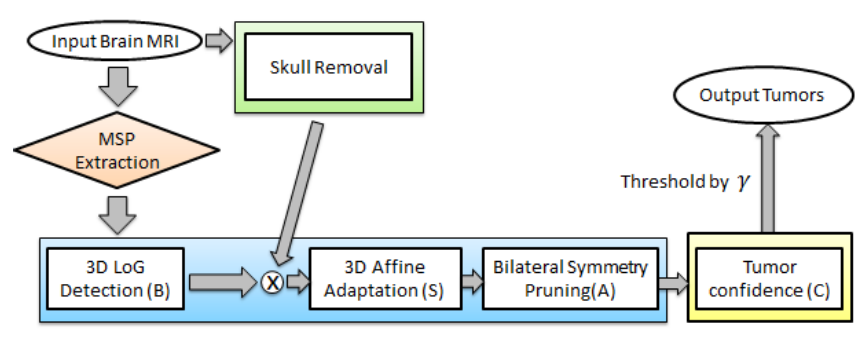

Fig. 2. The pruning sequence of the proposed method.

Using Eq. 3, the radius $r$ of each detected blob can be calculated as $r=\sqrt{3} \sigma$.

We apply 3D blob detection at 10 different scales, and normalize the detection responses at each scale to be comparable across scales. Since the LoG function sums to 0 and its center region sums to -1 , the normalizing factor $c(\sigma)$ for scale $\sigma$ can be found by integrating over the center region. Solving this integration using spherical polar coordinates:

$$
c(\sigma) * \int_{r=0}^{R} \int_{\theta=0}^{2 \pi} \int_{\phi=0}^{\pi} \Delta g_{x y z} r^{2} \sin (\phi) d \phi d \theta d r=-1
$$

the normalizing factor is derived to be $c(\sigma)=e^{\frac{3}{2}} \sigma^{2} / 3 \sqrt{\frac{6}{\pi}}$, which is proportional to $\sigma^{2}$. The strength (saliency) of blobs detected at different scales can therefore be compared fairly using the normalized blob detection response $B=\sigma^{2} * h(x, y, z \mid \sigma)$. A $5 \times 5 \times 5$ non-maximum suppression operator across scales is applied to the normalized 3D LoG detection responses $B$ to eliminate weak interest points.

\subsection{Affine Adaptation and Shape Pruning}

Besides tumors, the 3D LoG detector may pick up structures such as blood vessels, ventricles, and skull plates. Since these tend to be more elongated in shape, we prune the detected 3D blobs by applying 3D affine adaptation and discarding ones with highly elliptical shapes.

For every detected 3D blob, we find the overall gradient direction from the blob's enclosed boundary using its 3D structure tensor (second moment matrix) $\mathbf{M}$, where $\mathbf{M}=\mathbf{I}^{\prime} \mathbf{I}$ with $\mathbf{I}=\left[I_{x}, I_{y}, I_{z}\right]$ being a column vector containing the gradient information along the dimensions $x, y$, and $z$ :

$$
M=\left[\begin{array}{ccc}
I_{x}^{2} & I_{x} I_{y} & I_{x} I_{z} \\
I_{x} I_{y} & I_{y}^{2} & I_{y} I_{z} \\
I_{x} I_{z} & I_{y} I_{z} & I_{z}^{2}
\end{array}\right]
$$

Eigenvalue decomposition is applied to the structure tensor matrix $M$ to obtain eigenvalues $\left(\lambda_{1}, \lambda_{2}, \lambda_{3}\right)$ and eigenvectors $\left(\overrightarrow{u_{1}}, \overrightarrow{u_{2}}, \overrightarrow{u_{3}}\right)$. The eigenvalues represent the $3 \mathrm{D}$ elliptical shape of each 3D blob, and the eigenvectors give the ellipsoid axis orientations in 3D. The affine adapted shape score $S$ for 
A)
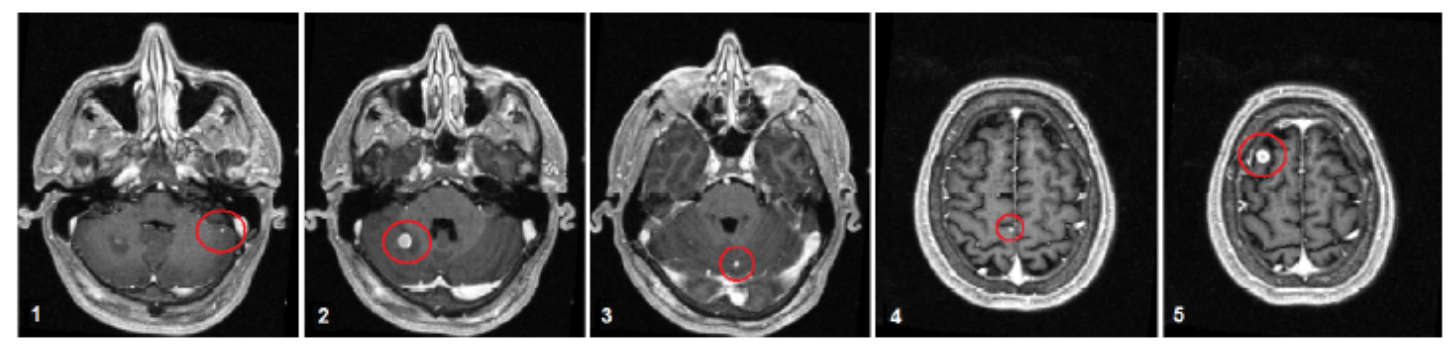

B)
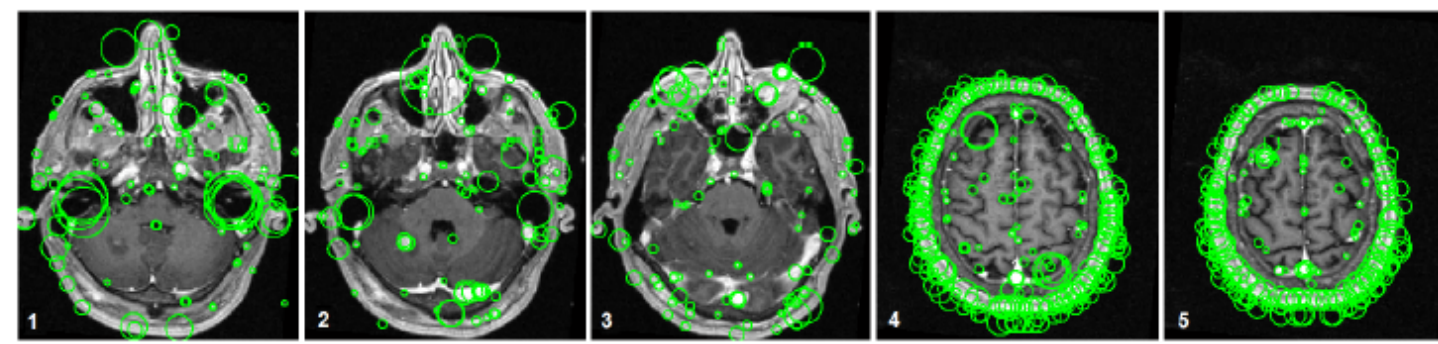

C)
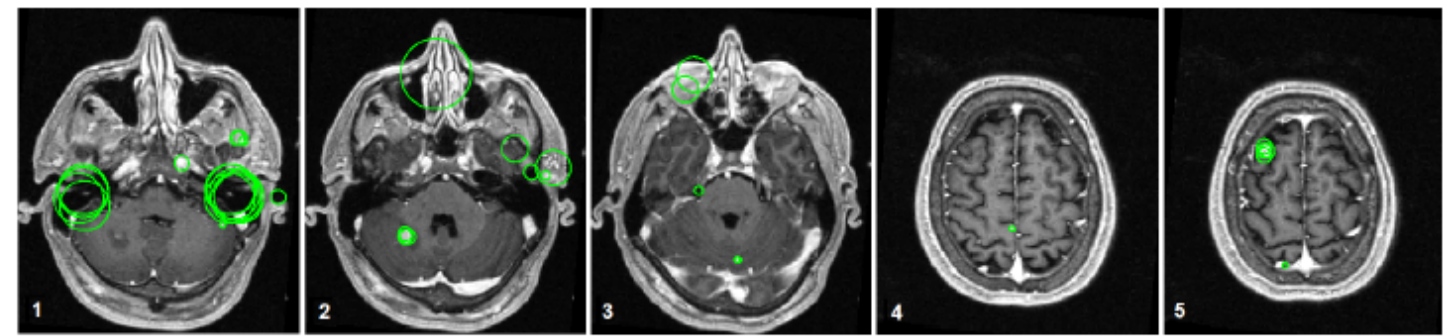

D)
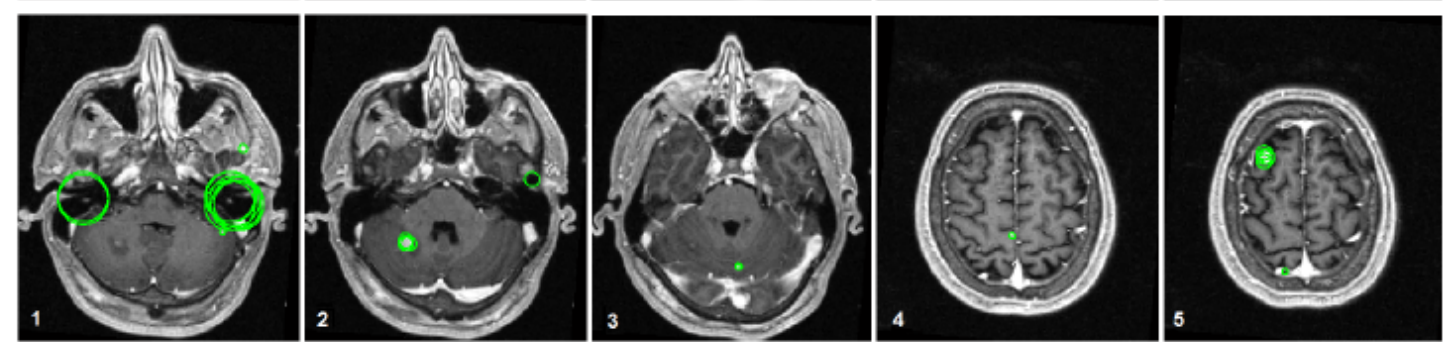

E)
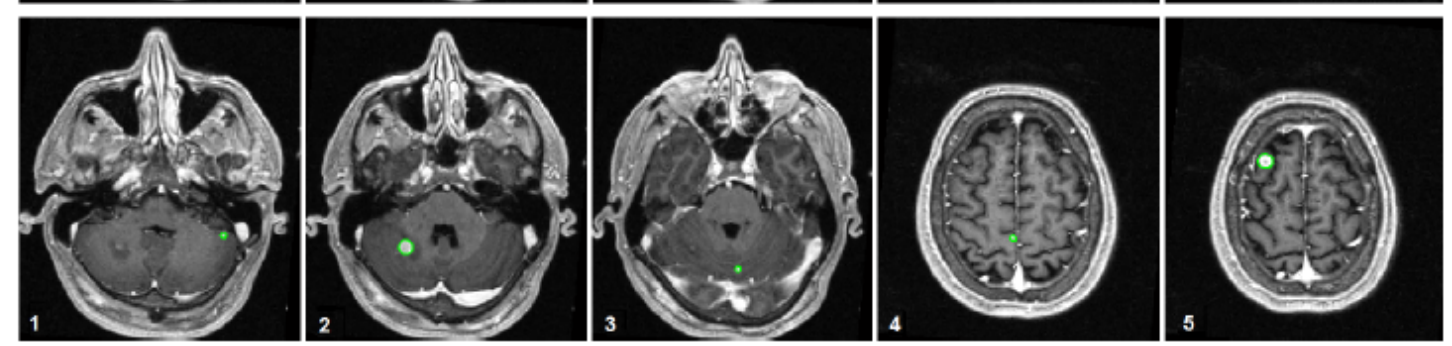

Fig. 3. Intermediate and final results for case \#17 in Table 1. A) representative 2D slides of the original 3D scan, five tumors are circled in red. B) initially detected 3D blobs, 27,561 total. C) after shape compactness pruning: 3,631 blobs remaining. D) after bilateral symmetry-based pruning: 1,452 blobs remaining. E) final tumor detection results, when the tumor likelihood score $C(B, S, A)$ is set at threshold $\gamma_{5}$ (Fig. 4B, Table 1): 11 blobs remaining. Best viewed in color.

use in Eq.11 is calculated as the length ratio of the shortest and longest axes:

$$
S=\frac{\min \left(\lambda_{1}, \lambda_{2}, \lambda_{3}\right)}{\max \left(\lambda_{1}, \lambda_{2}, \lambda_{3}\right)}
$$

\subsection{Bilateral Symmetry-based Pruning}

Normal human brains exhibit an approximate bilateral symmetry, while non-brain-stem tumors often break this symmetry. Therefore, blobs that have a bilateral match can be 


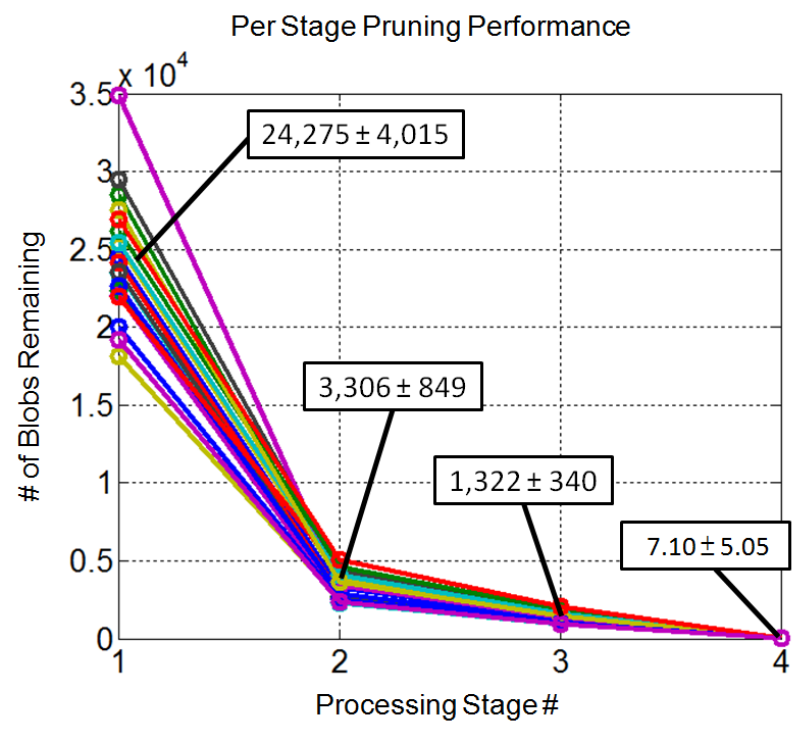

(A)

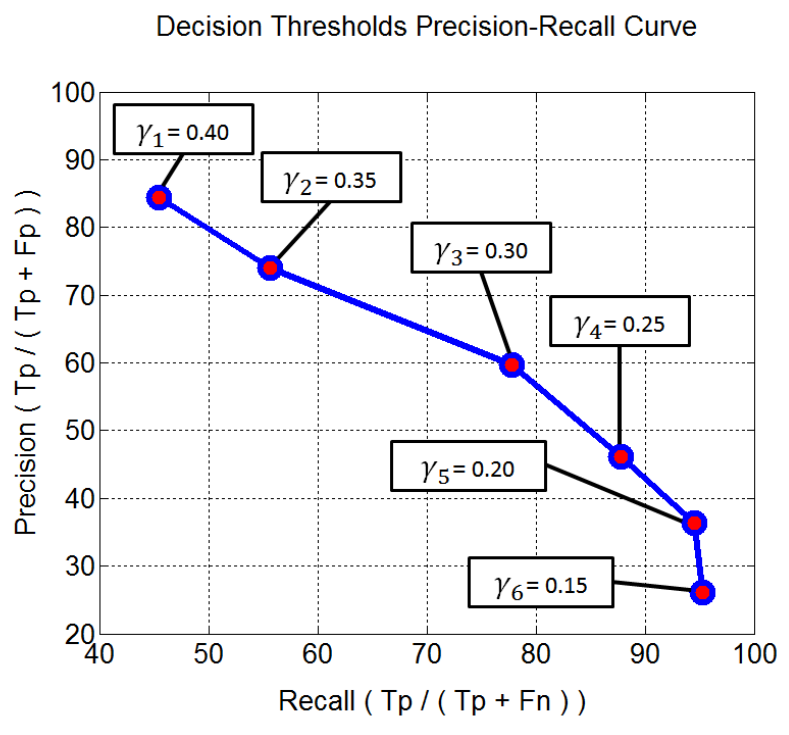

(B)

Fig. 4. (A): The cascade pruning results on twenty $3 D$ MR brain scans (Table 1 in terms of their individual (curves) and average number (mean \pm std) of 3D blobs at each processing stage (1: initially generated 3D blobs; 2: shape compactness $S$ pruning; 3: bilateral symmetry $A$ pruning; and 4: likelihood $C$ thresholding). (B): The algorithm performance shown as a precision-recall curve while varying the likelihood threshold $\left(\gamma_{1, \ldots, 6}\right)$ applied to $C$. The three thresholds that achieve the highest recall rates $\left(\gamma_{4,5,6}\right)$ are labeled in the plot and their detailed outcomes are shown in Table 1.

discarded as they are likely to be normal blob-like structures of the brain. To determine the level of asymmetry produced by a 3D blob, we compare the blob to its bilaterally symmetrical location with respect to the MSP. We use Earth Mover's Distance (EMD) as a metric to compare how similar the enclosed cumulative intensity distribution, $I(b)$, of a 3D blob is to that of its reflected location, $I(r e f(b))$. Note that both $I(b)$ and $I(r e f(b))$ are $1 \mathrm{D}$ (intensity) distributions, and that EMD and Mallow's distance in 1D are equivalent [16], where Mallow's distance between CDFs $x$ and $y$ is defined as $M(x, y)=1 / n \sum_{i=1}^{n}\left|x_{i}-y_{i}\right|$. This is simply the L1norm of two sorted vectors, which is computable in linear time. We define the asymmetry score $A$ of a given $3 \mathrm{D}$ blob as $A=M(I(b), I($ ref $(b)))$.

\section{EXPERIMENTS AND RESULTS}

We evaluate our method on 20 clinical 3D brain MR images with tumors and 5 normal brain scans provided by our medical collaborator. All tumors from the 20 pathological 3D MR images are identified by the same radiologist, and serve as the human-labeled ground truth for our validation. The 25 scans are of single T1 modality with gadolinium enhancement, acquired in the axial plane with $1 \mathrm{~mm}$ slice thickness into $256 \times 256 \times 256$ spatial resolution using a Philips Intera 1.5 Tesla Magnet scanner. Among the 20 pathological brains, there is a total of 85 tumors that are $2-38 \mathrm{~mm}$ in diameter, and
$3-28079 \mathrm{~mm}^{3}$ in volume with both homogeneous and heterogeneous necrotic cores. We perform the 3D LoG filtering at ten scales $\sigma=\{1,2,3,4,5,7,10,14\}$, which allows detection of tumors with radius approximately $1.7 \mathrm{~mm}$ to $24.2 \mathrm{~mm}$ ( $\sim 20.6$ to $59366 \mathrm{~mm}^{3}$ in volume). Table 1 presents quantitative results of our detection algorithm on the 20 pathological brain images. Interestingly, the method is able to detect actual tumors with smaller dimensions than the pre-set scales (cases $7,10,11,17,18$ have tumors with volume $<20 \mathrm{~mm}^{3}$ ). Fig. 3 illustrates the intermediate results of our sequential pruning method on sample case \#17, where all 5 metastatic brain tumors are detected including a tumor with a volume of $5 \mathrm{~mm}^{3}$.

Precision and recall rates are used as our performance measures. The precision rate is the ratio of detected blobs that are tumors over all detected blobs, while the recall rate is the ratio of detected tumors to all true tumors. We qualitatively consider a True Positive ( $T_{p}$ ) to be an extracted region that overlaps the vast majority ( $\geq 70 \%)$ of a tumor volume, a False Positive $\left(F_{p}\right)$ to be an extracted region that overlaps little to none of any part of the tumor volume, and a False Negative $\left(F_{n}\right)$ as a tumor region that is not part of any extracted blob. Precision is thus defined as $T_{p} /\left(T_{p}+F_{p}\right)$ and recall is defined as $T_{p} /\left(T_{p}+F_{n}\right)$.

Fig. $44 \mathrm{~A}$ shows the performance of our sequential falsepositives pruning process per stage (i.e. pruning by $S$, then $A$, then $C$ ), and Fig. $4 \mathrm{~B}$ shows a ROC curve for 6 different empirically chosen thresholds (denoted as $\gamma_{1, \ldots, 6}$ ) applied to $C$. 


\begin{tabular}{|c|c|c|c|c|c|c|c|c|c|c|c|}
\hline \multicolumn{3}{|c|}{ Case Information } & \multicolumn{3}{|c|}{ Tumor Volume $\left(\mathrm{mm}^{3}\right)$} & \multicolumn{6}{|c|}{ Results at Three Threshold Levels of $C$ (Tp / Total $\mid \mathrm{Fp})$} \\
\hline Case\# & Type & \# Tumors & Mean & Min & $\operatorname{Max}$ & $\gamma_{4}=$ & & $\gamma_{5}=$ & & & \\
\hline 1 & $\mathrm{M}$ & 1 & 12107 & 12107 & 12107 & $1 / 1$ & 2 & $1 / 1$ & 2 & $1 / 1$ & 6 \\
\hline 2 & $\mathrm{P}$ & 1 & 1448 & 1448 & 1448 & $1 / 1$ & 3 & $1 / 1$ & 9 & $1 / 1$ & 16 \\
\hline 3 & M & 2 & 10809 & 3532 & 18086 & $1 / 2$ & 2 & $2 / 2$ & 6 & $2 / 2$ & 8 \\
\hline 4 & M & 4 & 355.25 & 140 & 732 & $4 / 5$ & 2 & $4 / 5$ & 2 & $4 / 5$ & 3 \\
\hline 5 & M & 11 & 135.91 & 4 & 625 & $9 / 11$ & 6 & $9 / 11$ & 8 & $10 / 11$ & 11 \\
\hline 6 & $\mathrm{P}$ & 1 & 11913 & 11913 & 11913 & $1 / 1$ & 2 & $1 / 1$ & 5 & $1 / 1$ & 6 \\
\hline 7 & M & 15 & 82.31 & 3 & 422 & $12 / 15$ & 10 & $14 / 15$ & 15 & $15 / 15$ & 20 \\
\hline 8 & $P$ & 1 & 2262 & 2262 & 2262 & $1 / 1$ & 2 & $1 / 1$ & 5 & $1 / 1$ & 8 \\
\hline 9 & M & 1 & 3723 & 3723 & 3723 & $1 / 1$ & 6 & $1 / 1$ & 10 & $1 / 1$ & 12 \\
\hline 10 & M & 5 & 5879 & 5 & 28079 & $4 / 5$ & 2 & $5 / 5$ & 7 & $5 / 5$ & 8 \\
\hline 11 & M & 8 & 464.63 & 18 & 2226 & $8 / 8$ & 6 & $8 / 8$ & 6 & $8 / 8$ & 8 \\
\hline 12 & $\mathrm{P}$ & 1 & 12639 & 12639 & 12639 & $1 / 1$ & 4 & $1 / 1$ & 5 & $1 / 1$ & 10 \\
\hline 13 & M & 1 & 22033 & 22033 & 22033 & $1 / 1$ & 2 & $1 / 1$ & 6 & $1 / 1$ & 11 \\
\hline 14 & M & 1 & 150 & 150 & 150 & $1 / 1$ & 9 & $1 / 1$ & 15 & $1 / 1$ & 18 \\
\hline 15 & M & 2 & 10829 & 3617 & 18041 & $2 / 2$ & 3 & $2 / 2$ & 8 & $2 / 2$ & 14 \\
\hline 16 & M & 8 & 602.25 & 25 & 2145 & $4 / 8$ & 1 & $6 / 8$ & 5 & $6 / 8$ & 6 \\
\hline 17 & M & 5 & 192.40 & 12 & 533 & $5 / 5$ & 3 & $5 / 5$ & 6 & $5 / 5$ & 12 \\
\hline 18 & M & 10 & 572.90 & 9 & 3553 & $6 / 10$ & 3 & $6 / 10$ & 5 & $6 / 10$ & 9 \\
\hline 19 & M & 2 & 179.50 & 56 & 303 & $2 / 2$ & 0 & $2 / 2$ & 0 & $2 / 2$ & 3 \\
\hline 20 & M & 4 & 487.50 & 30 & 1426 & $3 / 4$ & 6 & $4 / 4$ & 12 & $4 / 4$ & 20 \\
\hline Total & - & - & - & - & - & $68 / 85$ & 74 & $75 / 85$ & 137 & $77 / 85$ & 209 \\
\hline Recall & - & - & - & - & - & $87.84 \pm$ & $.47 \%$ & $94.51 \pm$ & $.23 \%$ & 95.30 & $92 \%$ \\
\hline Precision & - & - & - & - & - & $46.17 \pm$ & $.44 \%$ & $35.71 \pm$ & $.18 \%$ & 26.03 & $78 \%$ \\
\hline Tp : Fp & - & - & - & - & - & $1:$ & & & & & \\
\hline Final Blob \# & - & - & - & - & - & $7.10_{=}$ & & 10.60 & 5.02 & 14.3 & 5.99 \\
\hline
\end{tabular}

Table 1. Automatic brain tumor detection results are shown at three threshold levels for $C$ with the highest recall rates $\left(\gamma_{4,5,6}\right.$, Fig. 4B). Case-Type $P$ and $M$ stand for Primary and Metastatic tumors respectively. The smallest volume of the detected tumor is $3 \mathrm{~mm}^{3}$ (case 5), and the largest is $28,079 \mathrm{~mm}^{3}$ (case 10).

The plot shows that the pruning stages are able to drastically reduce false positives from extremely high initial numbers $(\sim$ $24,000)$ to single digits, with a mean end-to-end running time of under 3 minutes per 3D brain MRI using a $2.7 \mathrm{Ghz}$ Intel Core 17 machine with $8 \mathrm{~Gb}$ RAM. The three thresholds that achieve the highest recall from Fig. $4 \mathrm{~B}\left(\gamma_{4,5,6}\right)$ are the ones reported in Table 1 . We also ran our algorithm on 5 normal brain scans. Table 2 shows that the final 3D blob count for the 5 normal brain subjects is lower, on average, than the count for subjects with tumors. That is, the average final blob count for normal brains is $0.8,6.4$, and 12.4 for the same 3 thresholds as yielded average blob counts of 7.1, 10.6 and 14.3 for the pathological brains in Table 1. This suggests that blob count can potentially be used as a discriminating feature to help classify between normal and pathological brains.

The affine-adapted ellipsoids over the final detected 3D blob already represent a good initial segmentation of the detected brain tumor in nearly all cases. For a more precise delineation, our detected blobs can act as automatic initialization regions for many state-of-the-art segmentation methods, such as the IFT-Watershed [17]. Fig. 5] shows the segmen-

\begin{tabular}{|c|c|c|c|}
\hline Normal & \multicolumn{3}{|c|}{ Results at 3 Threshold Levels (Fp) } \\
\hline Case & $\boldsymbol{\gamma}_{\mathbf{4}}$ & $\gamma_{5}$ & $\gamma_{6}$ \\
\hline 1 & $\mathbf{1}$ & 9 & 19 \\
2 & $\mathbf{1}$ & 2 & 8 \\
3 & $\mathbf{1}$ & 6 & 13 \\
4 & $\mathbf{0}$ & 11 & 17 \\
5 & $\mathbf{1}$ & 4 & 5 \\
\hline Avg \# of Blobs & $\mathbf{0 . 8 0} \pm \mathbf{0 . 4 5}$ & $6.40 \pm 3.65$ & $12.40 \pm 5.90$ \\
\hline
\end{tabular}

Table 2. On five normal brains, the final number of 3D blobs in each case is drastically smaller than that of pathological cases (Fig. 4A), in particular when the $C$ threshold is set to $\gamma_{4}$ (Fig. 4B).

tation result for sample case 15, which has a high Jaccard Coefficient $\left(T_{p} /\left(F_{p}+T_{p}+T_{n}\right)\right)$ of $90.14 \%$.

\section{CONCLUSION}

In this work, we have proposed a fast and fully automatic 3D brain tumor detection method using 3D blob detection, fol- 

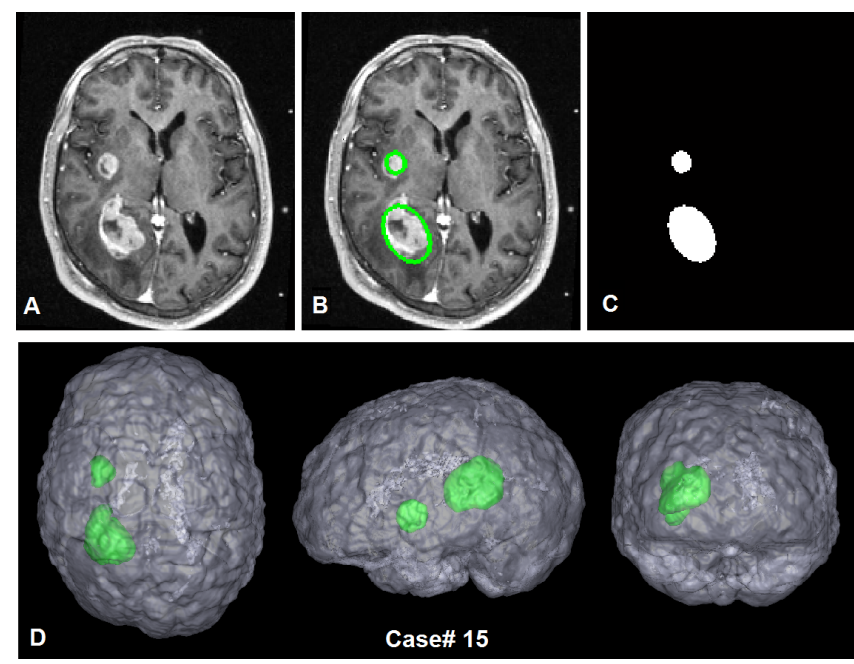

Fig. 5. A: case \#15 original image, showing 2 tumors in a representative slice; $\mathrm{B}$ : the detection result of our proposed method; C: the binary mask from the detected 3D blobs for automatic segmentation seeding; D: the 3D segmentation result, which has a Jaccard Coefficient of 90.14\%. See our supplemental movie for additional examples.

lowed by principles of shape compactness and asymmetry. Our use of the Laplacian of Gaussian to find 3D blobs is scaleinvariant and highly sensitive to small abnormalities (as small as $3 \mathrm{~mm}^{3}$ ). Subsequent affine adaptation and asymmetrybased pruning stages result in low false positive $F_{p}$ tumor detections. Our average $95.3 \%$ detection rate, average 3-10 false positives $F_{p}$ per brain (Table 1), and under 3 minute run-time (on a standard PC running Intel Core i7) are an improvement over state-of-the-art algorithms (average detection rate $90 \%$, average $F_{p}$ per brain 34.8 by [9][10], and 30 minutes using template matching in [10]). The detection results can also be applied to other brain abnormality detection tasks using the final 3D blobs as features. Finally, our detection results can be used as foreground seeds for automatic tumor delineation using any state-of-the-art segmentation method, and the number and salience of tumor hypotheses found may serve as potential discriminative measures between normal and pathological brains.

\section{ACKNOWLEDGEMENT}

This work is supported in part by a Grace Woodward grant for collaborative research in engineering and medicine at PSU (PI: Liu/Nguyen) and by the CNPq agency. We are grateful to Dr. R. Kikinis's lab for providing some of the brain scans.

\section{REFERENCES}

[1] R. Audigier and R. A. Lotufo, "Watershed by image foresting transform, tie-zone, and theoretical relationships with other watershed definitions," in International Symposium on Mathematical Morphology, 2007.

[2] P.A. Yushkevich, J. Piven, H.C. Hazlett, R.G. Smith, S. Ho, J.C. Gee, and G. Gerig, "User-guided 3D active contour segmentation of anatomical structures: Significantly improved efficiency and reliability," Neuroimage, 2006.

[3] J.J. Corso, E. Sharon, S. Dube, S. El-Saden, U. Sinha, and A. Yuille, "Efficient multilevel brain tumor segmentation with integrated bayesian model classification," TMI, 2008.

[4] D. Cobzas, N. Birkbeck, M. Schmidt, M. Jagersand, and A. Murtha, "3D variational brain tumor segmentation using a high dimensional feature set," in ICCV, 2007.

[5] C.H. Lee, S. Wang, A. Murtha, M.R.G. Brown, and R. Greiner, "Segmenting brain tumors using pseudo-conditional random fields," in MICCAI, 2008.

[6] C. Lee, R. Greiner, and M. Schmidt, "Support vector random fields for spatial classification," in PKDD, 2005.

[7] D. Koshy, C. Yu, D. T.D. Nguyen, S. Kashyap, R. T. Collins, and Y. Liu, "Supervised machine learning for brain tumor detection in structural MRI," Radiological Society of North America (RSNA), 2011.

[8] Sahar Ghanavati, Junning Li, Ting Liu, Paul S Babyn, Wendy Doda, and G Lampropoulos, "Automatic brain tumor detection in magnetic resonance images," in ISBI, 2012.

[9] T. Sugimoto, S. Katsuragawa, T. Hirai, R. Murakami, and Y. Yamashita, "Computerized detection of metastatic brain tumors on contrast-enhanced 3D MR images by using a selective enhancement filter," in World Congress on Medical Physics and Biomedical Engineering, 2010.

[10] R. Ambrosini and P. Wang, "Computer-aided detection of metastatic brain tumors using automated three-dimensional template matching," Journal of MRI, 2010.

[11] N. Ray, B. N. Saha, and M. Brown, "Locating brain tumors from MR imagery using symmetry," in Asilomar SSC, 2007.

[12] C. Yu, G. C.S. Ruppert, D. TD Nguyen, A. X. Falcao, and Y. Liu, "Statistical asymmetry-based brain tumor segmentation from 3D MR images.," in BIOSIGNALS, 2012.

[13] G. C. S. Ruppert, L. Teverovskiy, C. Yu, A. X. Falcao, and Y. Liu, "A new symmetry-based method for mid-sagittal plane extraction in neuroimages," in ISBI, 2011.

[14] S.M. Smith, "Fast robust automated brain extraction," Human Brain Mapping, 2002.

[15] Y. Liu, R. T. Collins, and W. E. Rothfus, Automatic extraction of the central symmetry (mid-sagittal) plane from neuroradiology images, Carnegie Mellon University, the Robotics Institute TR-96-40, 1996.

[16] E. Levina and P. Bickel, "The earth mover's distance is the mallows distance: Some insights from statistics," in ICCV, 2001.

[17] R. Lotufo and A. Falcao, "The ordered queue and the optimality of the watershed approaches," in Mathematical Morphology and its Applications to Image and Signal Processing, 2002. 